EESTI NSV TEADUSTE AKADEEMIA TOIMETISED. 29. KOIDE

FOOSIKA * MATEMAATIKA. 1980, NR. 3

ИЗВЕСТИЯ АКАДЕМИИ НАУК ЭСТОНСКОИ ССР. ТОМ 29

ФИЗИКА * МАТЕМАТИКА. 1980, № 3

П. ПУУСЕМП

удК $\quad 519.443$

\title{
ОБ ОПРЕДЕЛЯЕМОСТИ ПЕРИОДИЧЕСКОЙ АБЕЛЕВОЙ ГРУППЫ СВОЕЙ ПОЛУГРУППОЙ ЭНДОМОРФИЗМОВ
}

\author{
(ІІредставил А. Хумал)
}

1. В $\left[{ }^{1,2}\right]$ было показано, что каждая периодическая абелева группа определяется своим кольцом эндоморфизмов, т. е. изоморфизм колец эндоморфизмов двух периодических абелевых групп влечет за собой изоморфизм самих этих групп. В $\left[{ }^{3}\right]$ было доказано, что каждая конечная абелева группа $G$ определяется уже своей полугруппой всех эндоморфизмов End $G$ (в классе всех групп), т. е. из изоморфизма End $G \simeq$ $\simeq$ End $H$, где $H-$ некоторая другая группа (не обязательно абелева), следует изоморфизм групп $G$ и $H$. В настоящей работе этот результат обобщается и доказывается, что всякая периодическая абелева группа, порядки элементов которой ограничены в совокупности, определяется своей полугруппой всех эндоморфизмов.

Кроме общепринятых и упомянутых обозначений, будем придерживаться следующих: $Z(G)$ - центр группы $G ; g^{*}$ - внутренний автоморфизм, порожденный элементом $g ; \pi(G)$ - множество всех простых делителей порядков элементов периодической группы $G$; $\langle g\rangle-$ циклическая подгруппа, порожденная элементом $g$ группы $G ; \operatorname{Im} x-$ образ эндоморфизма $x$; $\operatorname{Ker} x$ - ядро эндоморфизма $x$.

2. Следуя $\left[{ }^{4}\right]$ (с. 303 ), назовем эндоморфизмы $x$ и $y$ группы $G$ суммируемыми, если $g h=h g$ для каждых $g \in \operatorname{Im} x$ и $h \in \operatorname{Im} y$. Отображение, переводящее всякий элемент $g \in G$ в элемент $(g x)(g y)$, называется в случае суммируемых эндоморфизмов суммой $x$ и $y$, т. $е$ $g(x+y)=(g x)(g y)$. Отображение $x+y$ будет эндоморфизмом группы $G$. Эндоморфизмы $x$ и $y$ будем называть ортогональными, если $x y=y x=0$.

В силу $\left[{ }^{3}\right]$ (с. 79,85 и 86) имеют место следующие две леммы.

Л ем м а 1. Если идемпотенты $x_{1}, \ldots, x_{n}$ полугруппы End $G$ попарноортогональны и суммируемь, то существует сумма $x_{1}+\ldots+x_{n}$, которая является тоже идемпотентом, и

$$
\begin{aligned}
\operatorname{Ker}\left(x_{1}+\ldots+x_{n}\right) & =\bigcap_{i=1}^{n} \operatorname{Ker} x_{i}, \quad \operatorname{Im}\left(x_{1}+\ldots+x_{n}\right)=\boldsymbol{\Pi}_{i=1}^{n} \operatorname{Im} x_{i}, \\
G & =\left(\bigcap_{i=1}^{n} \operatorname{Ker} x_{i}\right) \lambda\left(\boldsymbol{\Pi}_{i=1}^{n} \operatorname{Im} x_{i}\right) .
\end{aligned}
$$

Л ем м а 2. Если $\varphi:$ End $G \rightarrow$ End $H-$ изоморфизм (G и торые группы) $и x_{1}, \ldots, x_{n}$ - попарно-ортогональные и сумлируемье идемпотенты из End $G$, то идемпотенты $x_{1} \varphi, \ldots, x_{n} \varphi$ также попарноортогональны, суммируемы и

$$
\left(x_{1}+\ldots+x_{n}\right) \varphi=\left(x_{1} \varphi\right)+\ldots+\left(x_{n} \varphi\right) .
$$


3. Пусть группа $G=\boldsymbol{\Pi}_{i \in I} G_{i}$ является внутренним прямым произведением своих подгрупп $G_{i}, i \in I$. Обозначим через $\pi_{i}$ проекцию группы $G$ на ее подгруппу $G_{i}$. Предположим, что $H-$ некоторая другая группа, для которой End $G \simeq$ End $H$. Пусть $\varphi$ : End $G \rightarrow$ End $H-$ соответствующий изоморфизм и $\tau_{i}=\pi_{i} \varphi, H_{i}=\operatorname{Im} \tau_{i}=H \tau_{i} \quad(i \in I)$. Докажем при сделанных предположениях следующие леммы.

Л ем м а 3. Если $\tau \in$ End $H \quad$ u $\tau_{i} \tau=0_{H}$ при каждом $i \in I$, то $\tau=0_{H}$.

Действительно, тогда $0_{G}=0_{H} \varphi^{-1}=\left(\tau_{i} \tau\right) \varphi^{-1}=\left(\tau_{i} \varphi^{-1}\right)\left(\tau \varphi^{-1}\right)=$ $=\pi_{i}\left(\tau \varphi^{-1}\right) \quad$ и $\quad G_{i}\left(\tau \varphi^{-1}\right)=\left(G_{i} \pi_{i}\right)\left(\tau \varphi^{-1}\right)=G_{i}\left(\pi_{i}\left(\tau \varphi^{-1}\right)\right)=G_{i} 0_{G}=\{1\}$ при каждом $i \in I$. Следовательно, $\tau \varphi^{-1}=0_{G}$ и $\tau=0_{G} \varphi=0_{H}$.

Лемма 4. Для каждого $i \in I$ имеет место разложение $H=$ $=\operatorname{Ker} \tau_{i} \times \operatorname{Im} \tau_{i}=\operatorname{Ker} \tau_{i} \times H_{i}$.

Доказательство. Пусть $i \in I$. Обозначим через $\pi_{i}^{*}$ проекцию группы $G$ на еe подгруппу $\boldsymbol{\Pi}_{j \in I \backslash\{i\}} G_{j}$. Тогда идемпотенты $\pi_{i}$ и $\pi_{i}{ }^{*}$ полугруппы End $G$ ортогональны, суммируемы и $\pi_{i}+\pi_{i}^{*}=1_{G}$. По лемме 2 идемпотенты $\tau_{i}=\pi_{i} \varphi$ и $\pi_{i}^{*} \varphi$ полугруппы End $H$ также ортогональны, суммируемы и $\left(\pi_{i}+\pi_{i}{ }^{*}\right) \varphi=\pi_{i} \varphi+\pi_{i}{ }^{*} \varphi$. Поэтому $\tau_{i}+\pi_{i}^{*} \varphi=1_{H}$ и по лемме 1 имеем $H=\operatorname{Im~} 1_{H}=\operatorname{Im} \tau_{i} \times \operatorname{Im}\left(\pi_{i}^{*} \varphi\right)$. Ввиду ортогональности идемпотентов $\tau_{i}$ и $\pi_{i}{ }^{*} \varphi$ ясно, что $\operatorname{Im}\left(\pi_{i}{ }^{*} \varphi\right)=$ $=\operatorname{Ker} \tau_{i}$. Следовательно, $H=\operatorname{Im} \tau_{i} \times \operatorname{Ker} \tau_{i}$. Лемма доказана.

Л емм а 5. Подгруппьь $K=\bigcap_{i \in I} \operatorname{Ker} \tau_{i} u H_{i}, i \in I$, порождают в группе Н прямое произведение

$$
K \times \boldsymbol{\Pi}_{i \in I} H_{i}=\left(\bigcap_{i \in I} \operatorname{Ker} \tau_{i}\right) \times\left(\boldsymbol{\Pi}_{i \in I} H_{i}\right) .
$$

Дока з а тельство. Пусть $1,2, \ldots, n-$ произвольный конечный набор различных индексов из множества $I$. Тогда проекции $\pi_{1}, \ldots, \pi_{n}$ попарно-ортогональны и суммируемы. По лемме 2 идемпотенты $\tau_{1}, \ldots, \tau_{n}$ полугруппы End $H$ тоже ортогональны, суммнруемы и в силу леммы 1

$$
\begin{gathered}
\operatorname{Im}\left(\tau_{1}+\ldots+\tau_{n}\right)=\boldsymbol{\Pi}_{k=1}^{n} \operatorname{Im} \tau_{k}=\Pi_{k=1}^{n} H_{k}, \\
\operatorname{Ker}\left(\tau_{1}+\ldots+\tau_{n}\right)=\bigcap_{k=1}^{n} \operatorname{Ker} \tau_{k} .
\end{gathered}
$$

В силу произвольности выбора числа $n$ и набора $1, \ldots, n$ из равенства (2) следует, что подгруппы $H_{i}, i \in I$, порождают в группе $H$ прямое произведение $\boldsymbol{\Pi}_{i \in I} H_{i}$.

Предположим, что $g \in\left(\bigcap_{i \in I} \operatorname{Ker} \tau_{i}\right) \cap\left(\boldsymbol{\Pi}_{i \in I} H_{i}\right)$. Тогда существуют такие $1, \ldots, n \in I$, что $g \in\left(\bigcap_{k=1}^{n} \operatorname{Ker} \tau_{k}\right) \cap\left(\boldsymbol{\Pi}_{k=1}^{n} H_{k}\right)$, т. е. $g \in \operatorname{Ker}\left(\tau_{1} \leftarrow \ldots\right.$ $\left.\ldots+\tau_{n}\right) \cap \operatorname{Im}\left(\tau_{1}+\ldots+\tau_{n}\right)$. Так как $\tau_{1}+\ldots+\tau_{n}$ является идемпотентом и $\operatorname{Ker} \tau \cap \operatorname{Im} \tau=\{1\}$ для каждого идемпотентного эндоморфизма группы $H$, то $g=1$ и, следовательно,

$$
\left(\bigcap_{i \in I} \operatorname{Ker} \tau_{i}\right) \cap\left(\boldsymbol{I}_{i \in I} H_{i}\right)=\{1\} .
$$

В силу леммы 4 ясно, что каждый элемент группы $\bigcap_{i \in I} \operatorname{Ker} \tau_{i}$ перестановочен с каждым элементом группы $\boldsymbol{\Pi}_{i \in I} H_{i}$. Поэтому из равенства (3) следует равенство (1). Лемма доказана.

Обозначим

$$
M=K \times \boldsymbol{\Pi}_{i \in I} H_{i}=\left(\bigcap_{i \in I} \operatorname{Ker} \tau_{i}\right) \times\left(\boldsymbol{\Pi}_{i \in I} H_{i}\right) .
$$


Л емм а 6. Eсли группы $G_{i}$ конечны и циккличны $(i \in I)$, то $H_{i} \simeq G_{i}$ $(i \in I)$ и группа Н коммутативна.

Доказательство. Пусть выполнены предположения леммы. Из изоморфизма End $G \simeq$ End $H$ следует, в силу $\left[{ }^{3}\right]$ (лемма 1.6), изоморфизм End $\left(\operatorname{Im} \pi_{i}\right) \simeq$ End $\left(\operatorname{Im} \tau_{i}\right)$, т. е. End $G_{i} \simeq$ End $H_{i}(i \in I)$. Так как каждая конечная абелева группа определяется своей полугруппой эндоморфизмов $\left[{ }^{3}\right]$ (теорема 4.2$)$, то $G_{i} \simeq H_{i}(i \in I)$ и первое утверждение леммы доказано.

Для доказательства второго утверждения покажем сначала, что $M \subset Z(H)$. Пусть $g \in M$. Поскольку группы $H_{i}, i \in I$, цикличны, то в силу разложения (1) и ортогональности идемпотентов $\tau_{i}$ ясно, что $\tau_{i} g^{*}=\tau_{i} \quad$ при каждом $\quad i \in I$. Поэтому $\quad\left(\tau_{i} g^{*}\right) \varphi^{-1}=\tau_{i} \varphi^{-1}$, т. $\quad$ е. $\pi_{i}\left(g \varphi^{-1}\right)=\pi_{i}$ при каждом $i \in I$. Если теперь $b \in G_{i}$, то $b=b \pi_{i}=$ $=b\left(\pi_{i}\left(g^{*} \varphi^{-1}\right)\right)=\left(b \pi_{i}\right)\left(g^{*} \varphi^{-1}\right)=b\left(g^{*} \varphi^{-1}\right)$. Следовательно, $g^{*} \varphi^{-1} \mid G_{i}=$ $=1_{G} \mid G_{i}$ при каждом $i \in I$, т. е. $g^{*} \varphi^{-1}=1_{G}$ и $g^{*}=1_{H}$. Последнее равенство означает, что $g \in Z(H)$. Отсюда следует включение $M \subset Z(H)$.

Пусть $g \in H$. Тогда $\tau_{i} g^{*}=\tau_{i}(i \in I)$, ибо $M \subset Z(H)$. Теперь, по аналогии, $g \in Z(H)$. Следовательно, $H=Z(H)$ и группа $H$ коммутативна. Лемма доказана.

Лемм а 7. Если группь $G_{i}(i \in I)$ цикличны и порядки элементов группы $G$ ограничены в совокупности, то порядки элементов группы $H$ также ограничены в совокупности.

Д ок аз а тельство. По предположению леммы существует такое натуральное число $n$, что $g^{n}=1$ для каждого $g \in G$. В силу изоморфизма $G_{i} \simeq H_{i}(i \in I)$ при каждом $g \in \boldsymbol{I}_{i \in I} H_{i}$ выполняется равенство $g^{n}=1$. Поэтому отображение $\tau: g \rightarrow g^{n}, g \in H$, удовлетворяет равенствам $\tau_{i} \tau=0_{H}, i \in I$. Так как группа $H$ абелева в силу леммы 6 , то $\tau \in$ End $H$. В силу леммы $3 \tau=0_{H}, \quad$ т. е. $g^{n}=1$ для каждого $g \in H$. Лемма доказана.

Т еорем а. Всякая периодическая абелева группа, порядки элементов которой ограничены в совокупности, определяется своей полугруппой всех эндоморфизмов в классе всех групп.

Доказ а тельство. Пусть $G$ - абелева группа, порядки элементов которой ограничены в совокупности. Тогда группа $G$ разлагается на внутреннее прямое произведение своих циклических подгрупп $G_{i}$, $i \in I$ (см. [ $\left.{ }^{5}\right]$, с. 107). Можно считать, что порядки подгрупп $G_{i}$ являются степенями простых чисел.

Предположим, что полугруппы End $G$ и End $H$, где $H-$ некоторая другая группа, изоморфны. Покажем, что тогда группы $G$ и $H$ изоморфны. Будем предполагать, что $\varphi, H_{i}, \tau_{i}$ и $M$ имеют свои прежние значения.

По лемме 7 порядки элементов группы $H$ ограничены в совокупности, а по лемме 6 группа $H$ является абелевой. Поэтому порядки элементов группы $H / M$ также ограничены в совокупности и группа $H / M$ разлагается на прямое произведение своих циклических подгрупп:

$$
H / M=\boldsymbol{I}_{j \in J}\left\langle g_{j} M\right\rangle .
$$

Можно считать, что это разложение таково, что порядки элементов $g_{j}$ являются степенями простых чисел.

Покажем, что $\pi(M) \eta \pi(H / M)=\varnothing$. От противного предположим, что $\pi(M) \cap \pi(H / M) \neq \varnothing$, и пусть $p$ принадлежит данному пересече- 
нию. Тогда группа $M$ содержит элемент $c$ порядка $p$ и существует такой $j_{0} \in J$, что порядок өлемента $g_{j_{0}}$ является степенью простого числа $p$. Теперь можно построить следующую последовательность гомоморфизмов:

$$
H \stackrel{\varepsilon}{\rightarrow} H / M=\boldsymbol{\Pi}_{j \in J}\left\langle g_{j} M \stackrel{\alpha}{\rightarrow}_{\rightarrow}^{g_{j_{0}} M} \stackrel{\beta}{\rightarrow}\langle c\rangle,\right.
$$

где $\varepsilon-$ естественный гомоморфизм, $\alpha-$ проекция, $\left(g_{j_{0}} M\right) \beta=c$. Обозначим $\tau=\varepsilon \alpha \beta$. Тогда $\tau \in$ End $H$. Так как $H \tau_{i}=\operatorname{Im} \tau_{i}=H_{i} \subset M \subset$ $\subset \operatorname{Ker} \tau(i \in I)$, то $\tau_{i} \tau=0_{H}$ при каждом $i \in I$. По лемме $3 \tau=0_{H}$. Но по построению $\tau \neq 0_{H}$. Полученное противоречие показывает, что

$$
\pi(M) \cap \pi(H / M)=\varnothing .
$$

Из равенств (5) и (6) следует теперь разложение $H=$ $=\left(\boldsymbol{\Pi}_{j \in J}\left\langle g_{j}\right\rangle\right) \times M, \quad$ а из равенства $(4)-$ разложение

$$
H=K \times\left(\boldsymbol{\Pi}_{i \in I} H_{i}\right) \times\left(\boldsymbol{\Pi}_{j \in J}\left\langle g_{j}\right\rangle\right) .
$$

Обозначим через $\mu$ проекцию группы $H$ на ее подгруппу $K \times\left(\boldsymbol{\Pi}_{j \in J}\left\langle g_{j}\right\rangle\right)$. Тогда $\mu \in$ End $H \quad$ и $H \tau_{i}=H_{i} \subset \operatorname{Ker} \mu$, т. е. $\tau_{i} \mu=0_{H}$ при каждом $i \in I$. По лемме $3 \mu=0_{H}$. Следовательно, $K \times\left(\boldsymbol{\Pi}_{j \in J}\left\langle g_{j}\right\rangle\right)=\{1\}$ и $H=\boldsymbol{\Pi}_{i \in I} H_{i}$. Так как в силу леммы 6 группы $G_{i}$ и $H_{i}$ изоморфны $(i \in I)$, то группы $G$ и $H$ изоморфны. Теорема доказана.

\section{Л ИТЕ Р А Т Р А}

1. B a e r, R., Ann. Math., 44, № 2, 192-227 (1943).

2. K a p l a n s ky, I., Proc. Nat. Acad. Sci. USA, 38, $538-540$ (1952).

3. П у у с е м п П., Уч. зап. Тартуск. ун-та, № 366, 76-104 (1975).

4. К у р ош А. Г., Теория групп, М., «Наука», 1967.

5. Ф у к с Л., Бесконечные абелевы группы, т. 1, М., «Мир», 1974.

Таллинский политехнический институт

Поступила в редакцию 27/III 1980

\section{P. PUUSEMP}

\section{PERIOODILISE ABELI RUHMA MAARATAVUSEST OMA ENDOMORFISMI- POOLRUHMA ABIL}

Artiklis on käsitletud järgmist probleemi: millal isomorfismist End $A \simeq$ End $B$ (End $A-$ rühma $A$ kõigi endomorfismide poolrühm) järeldub rühmade $A$ ja $B$ isomorfism $A \simeq B$. Oldjuhul ei ole sellele küsimusele vastust, mōningaid tulemusi on avaldatud artiklites $\left[{ }^{1-3}\right]$. Näiteks on artiklis $\left[{ }^{3}\right]$ tõestatud järgmine tulemus: isomorfismist End $A \simeq$ End $B$, kus $A$ on lōplik Abeli rühm, järeldub isomorfism $A \simeq B$. Käesolevas artiklis on näidatud, et isomorfismist End $A \simeq$ End $B$, kus $A$ on perioodiline Abeli rühm, mille elementide järgud on tōkestatud, järeldub isomorfism $A \simeq B$. 


\section{P. PUUSEMP \\ 世. \\ ON THE DETERMINITY OF A PERIODIC ABELIAN GROUP BY ITS SEMIGROUP OF ENDOMORPHISMS}

Let a group $A$ be fixed and let End $A$ denote the semigroup of all endomorphisms of the group $A$. If from the isomorphism of semigroups End $A$ and End $B$ follows the isomorphism of groups $A$ and $B$, then we say that the group $A$ is determined by its semigroup of endomorphisms. In general, the necessary and sufficient conditions for determinity of an arbitrary group by its semigroup of endomorphisms are unknown. Some results on this problem are presented in the papers $\left[{ }^{1-3}\right]$. For example, in the paper $\left[^{3}\right]$ it is shown, that from the direct decomposition $A=A_{1} \times \ldots \times A_{n}$ and the isomorphism End $A \simeq$ End $B$ follows the direct decomposition $B=B_{1} \times \ldots \times B_{n}$ with a property End $A_{i} \simeq$ End $B_{i}$ for any $i \in\{1, \ldots, n\}$. From this result it follows simply that an arbitrary finite abelian group can be determined by its semigroup of endomorphisms (see $\left[{ }^{3}\right]$, theorem 4.2 ). In this paper, using the methods of the paper $\left[{ }^{3}\right]$, the last result is generalized. It is shown that from the isomorphism End $A \simeq$ End $B$, where $A$ is a periodic abelian group with bounded orders of its elements, follows the isomorphism of groups $A$ and $B$.

It should be noted that in great many papers a similar problem for rings of all endomorphisms of abelian groups is considered. For example, it is well known that from the isomorphism of rings of all endomorphisms of two periodic abelian groups $A$ and $B$ follows the isomorphism of groups $A$ and $B$. 